\title{
Analytical Solutions of the Diffusive Heat Equation as the Application for Multi-cellular Device Modeling - A Numerical Aspect
}

\author{
Zbigniew Lisik ${ }^{1}$, Janusz Wozny ${ }^{1}$, Malgorzata Langer ${ }^{1}$, and Niccolò Rinaldi ${ }^{2}$ \\ ${ }^{1}$ Department of Electronics and Telecommunications Engineering, \\ Technical University Of Lodz, 90-924 Lodz, ul. Wolcza ska 223, Poland, \\ jwozny@p. lodz.pl \\ ${ }^{2}$ Department of Electronics Engineering, University of Naples, „Federico II”, \\ Via Claudio 21, 50125 Naples, Italy
}

\begin{abstract}
This work concerns the thermal analysis of solid state devices, namely the solution of the heat transfer problem as part of the coupled electrical-thermal analysis of an IC. We investigate some typical numerical problems which can be found during evaluating of the time dependent solution of the heat diffusion equation for a 3D finite domain with an uniform heat source. Considering a surface heat source, the solutions obtained by means of the Large Time Greens Function and Small Time Greens Function are studied. A simple method to improve the computational efficiency is proposed. A comparison of different approaches is given as well.
\end{abstract}

\section{Introduction}

Power dissipation is one of the main problems in the development of ICs, due to the tremendous growth in both integration level and operation speed. Thermal effects can not be neglected in IC design since the phenomenon of the heat conduction markedly affects the electrical behavior of devices. Thermal coupling and self-heating effects should be included. Due to the interdependence of electrical and thermal effects, a coupled electro-thermal analysis is needed. The heat conduction is a $3 \mathrm{D}$ phenomenon in nature and any reduction in dimensions may lead to some considerable discrepancies compared to full 3D solution [1].

In any IC, heat dissipating elements comprise single devices or their clusters. Since the thickness of the IC active area is negligibly small in comparison with the substrate, the heat dissipating devices (active cells) can be treated as flat heat sources placed on the top surface of the substrate. In modern ICs, the density of such active cells can reach several millions. The temperature of each active cell can be treated as homogeneous due to very small cell size whereas its magnitude will depend both on the selfheating in the cell and on thermal coupling between adjacent cells. Such an approach to electrothermal simulation allows developing a procedure, in which the electrical and thermal models are split [5]. The electrical part is a combination of temperaturesensitive electrical models representing particular active cells that are treated as points characterized by one temperature, e.g. [5], whereas the thermal model is fully 3D, based on the solution of heat transport equation. 
The devices belonged to one active cell are treated as a point from "electrical" point of view or as the surface heat source from the "thermal" point of view. We will concentrate on its thermal counterpart. It is aimed at the evaluation of 3-D temperature distribution in the whole chip. It can be done by solving the heat transfer differential equation using a numerical or analytical approach. In the case of $3 \mathrm{D}$ domain representing the whole IC chip with several heat sources, the numerical approach would require extremely large computational effort giving the temperature distribution in a huge number of nodes whereas its value an a very few points is needed. These values could be obtained much easier from analytical solution if such a solution is available. It is possible in the case of considered thermal model but unfortunately, the analytical expressions have usually the form of infinite series. The number of elements in series must be limited in the computation process if it is to take a reasonable time. It is a crucial problem in the analytical thermal models and therefore it was the subject of consideration in this work. Different forms of the analytical formulas suitable for the 3D heat transport model in IC are investigated with respect to numerical efficiency of the calculation process.

\section{The Approach to the Thermal Analysis of IC}

Without loosing the generality of the approach, the IC is considered as the chip shown in Fig. 1a with active cells represented by $\mathrm{N}$ surface heat sources (SHS) with uniform heat generation whose magnitude is determined in the electrical model. Assuming that the system is linear, each source can be treated separately and the temperature at each point can be found as the superposition of temperature rises at the point caused by the different heat sources. The temperature contributions caused by the individual heat sources are given by the solutions obtained for the domain shown in Fig. 1b that includes the whole chip and one heat source only.
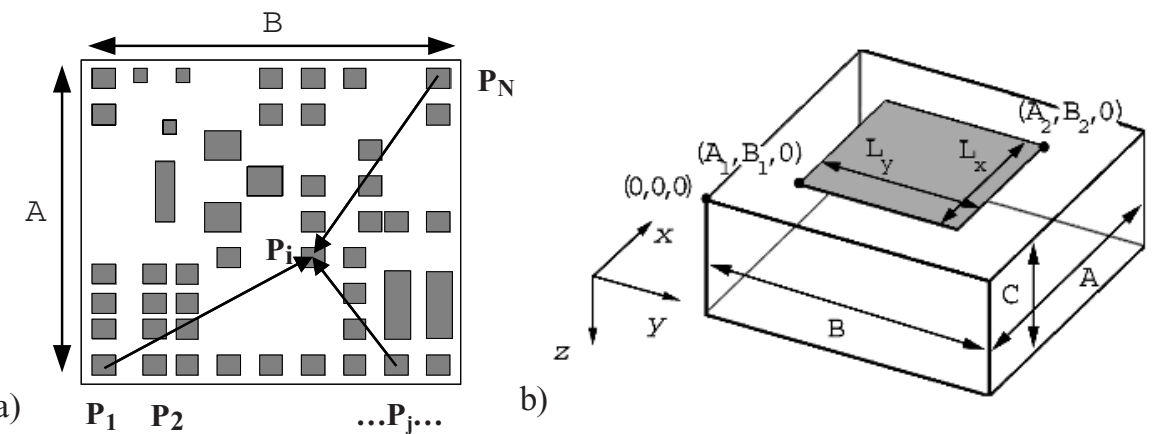

Fig. 1. a) The top surface of a IC with $\mathrm{N}$ heat sources b) A domain with a single source

The silicon homogeneous parallelepiped domain (Fig.1a) will be considered for the development of the analytical solutions. These have been obtained for the geometry in Fig. $1 \mathrm{~b}$ under the assumptions that the top and side walls are adiabatic and the bottom one, in contact with an ideal heat sink, is isothermal with a constant temperature $T_{h s}$ 
equal to the initial temperature $\mathrm{T}_{0}$. If we denote the heat generation density $\mathrm{g}(\mathrm{r}, \mathrm{t})$ as $\mathrm{g}(\mathrm{r}, \mathrm{t})=\mathrm{g}_{0 \mathrm{~S}}$, and the temperature $\mathrm{T}$ at a point $\mathrm{r}$ as:

$$
\mathrm{T}(\mathrm{r}, \mathrm{t})=\mathrm{T}_{\mathrm{hs}}+\theta(\mathrm{r}, \mathrm{t})
$$

The system of equations that has to be solved is as follows:

$$
\left\{\begin{array}{l}
\nabla^{2} \theta(\mathrm{r}, \mathrm{t})-\frac{1}{\alpha} \partial_{\mathrm{t}} \theta(\mathrm{r}, \mathrm{t})=-\frac{\mathrm{g}(\mathrm{r}, \mathrm{t})}{\mathrm{k}} \\
\left.\partial_{\mathrm{x}} \theta(\mathrm{r}, \mathrm{t})\right|_{\mathrm{x}=0, \mathrm{~A}}=\left.\partial_{\mathrm{y}} \theta(\mathrm{r}, \mathrm{t})\right|_{\mathrm{y}=0, \mathrm{~B}}=\left.\partial_{\mathrm{z}} \theta(\mathrm{r}, \mathrm{t})\right|_{\mathrm{z}=0}=0 \\
\left.\theta(\mathrm{r}, \mathrm{t})\right|_{\mathrm{z}=\mathrm{C}}=0, \quad \theta(\mathrm{x}, \mathrm{y}, \mathrm{z}, \mathrm{t}=0)=0
\end{array}\right.
$$

The $\mathrm{k}$ and $\alpha$ are the thermal conductivity and diffusivity, respectively. Since the thermal conductivity strongly depends on the temperature, in order to linearize the differential equation, the Kirchhoff transformation over k [3] can be always employed.

The solution of (2) is in a form of continuous function that can determine temperature for each place in the active cell. Since the electrical model requires one temperature for the whole cell only, one considered as the cell temperature that one calculated for the cell center. This way the final $i$-th cell temperature is determined by the sum:

$$
\mathrm{T}\left(\mathrm{r}_{\mathrm{c}, \mathrm{i}}, \mathrm{t}\right)=\mathrm{T}_{\mathrm{hs}}+\theta_{1}\left(\mathrm{r}_{\mathrm{c}, \mathrm{i}}, \mathrm{t}\right)+\ldots+\theta_{\mathrm{i}}\left(\mathrm{r}_{\mathrm{c}, \mathrm{i}}, \mathrm{t}\right)+\ldots+\theta_{\mathrm{N}}\left(\mathrm{r}_{\mathrm{c}, \mathrm{i}}, \mathrm{t}\right)
$$

where $r_{c, i}$ is the $i$-th cell center coordinate, $\theta_{j}\left(r_{c, i}, t\right)$ is the temperature increase in ith cell caused by the heat dissipation in $j$-th cell. If the mutual thermal resistance $R_{t h}$ $\mathrm{j}, \mathrm{i}\left(\mathrm{r}_{\mathrm{c}, \mathrm{i}}\right)$ in $\mathrm{i}$-th cell with respect to $\mathrm{j}$-th cells is defined as follows:

$$
\mathrm{R}_{\text {th } \mathrm{j}, \mathrm{i}}\left(\mathrm{r}_{\mathrm{c}, \mathrm{i}}\right)=\theta_{\mathrm{j}}\left(\mathrm{r}_{\mathrm{c}, \mathrm{i}}\right) / \mathrm{P}_{\mathrm{j}}
$$

the temperature at the i-th cell can be rewritten in the form, eg. [2,3]:

$$
\mathrm{T}\left(\mathrm{r}_{\mathrm{c}, \mathrm{i}}\right)=\mathrm{T}_{\mathrm{hs}}+\mathrm{P}_{1} \cdot \mathrm{R}_{\text {th } 1, \mathrm{i}}\left(\mathrm{r}_{\mathrm{c}, \mathrm{i}}\right)+\ldots+\mathrm{P}_{\mathrm{i}} \cdot \mathrm{R}_{\text {th } \mathrm{i}, \mathrm{i}}\left(\mathrm{r}_{\mathrm{c}, \mathrm{i}}\right)+\ldots+\mathrm{P}_{\mathrm{N}} \cdot \mathrm{R}_{\text {th } \mathrm{N}, \mathrm{i}}\left(\mathrm{r}_{\mathrm{c}, \mathrm{i}}\right)
$$

where $\mathrm{P}_{\mathrm{j}}$ power dissipated in $\mathrm{j}$-th cell. Then the temperature for a single active area is characterized by $\mathrm{N}$ thermal resistances. Eq. (5) describes the temperature in one active cell whereas the thermal model is to evaluate the temperatures in all the cells determined by $\mathrm{N}$ such equations.

If the transient case is considered, the thermal resistance $\mathrm{R}_{\mathrm{thj}, \mathrm{i}}$ is replaced by the thermal impedances $\mathrm{Z}_{\mathrm{thj}, \mathrm{i}}\left(\mathrm{r}_{\mathrm{c}, \mathrm{i}}, \mathrm{t}\right)$. The thermal impedance contains all necessary information to describe the thermal properties of a device.

\section{Analytical Solutions}

Usually, one can solve the same problem using different approaches that leads to different forms of its analytical solution. Let consider two such solutions of the problem described by (2); namely (6) and (7) called the Large Time Greens Function solution 
(LTGF) [1,4] and the Small Time Greens Function Solution (STGF) [4,6] (known also as the image method approach), respectively. The both are in a form of complex series but the difference between the formulas are so large that one can expected that their application in the considered thermal model cannot be equivalent from the point of view both computation effort and computation complexity.

$$
\begin{aligned}
& \mathrm{Z}_{\text {th }, \mathrm{LTGF}}(\mathrm{r}, \mathrm{t})=\frac{2}{\mathrm{kABCL}_{\mathrm{x}} \mathrm{L}_{\mathrm{y}}}\left\{\sum_{\mathrm{p}=1}^{\infty} \frac{\mathrm{L}_{\mathrm{x}} \mathrm{L}_{\mathrm{y}} \cos \left(v_{\mathrm{p}} \mathrm{z}\right)}{v_{\mathrm{p}}{ }^{2}}\left(1-\mathrm{e}^{-\alpha v_{\mathrm{p}}^{2} \mathrm{t}}\right)+\right. \\
& 2 \sum_{\mathrm{p}=1}^{\infty} \sum_{\mathrm{m}=1}^{\infty} \frac{\mathrm{L}_{\mathrm{Y}} \mathrm{J}_{\mathrm{m}} \cos \left(\beta_{\mathrm{m}} \mathrm{x}\right) \cos \left(v_{\mathrm{p}} \mathrm{z}\right)}{\beta_{\mathrm{m}}\left(\beta_{\mathrm{m}}{ }^{2}+v_{\mathrm{p}}{ }^{2}\right)}\left(1-\mathrm{e}^{-\alpha\left(\beta_{\mathrm{m}}^{2}+v_{\mathrm{p}}^{2}\right) \mathrm{t}}\right)+ \\
& 2 \sum_{\mathrm{p}=1}^{\infty} \sum_{\mathrm{n}=1}^{\infty} \frac{\mathrm{L}_{\mathrm{X}} \mathrm{K}_{\mathrm{n}} \cos \left(\gamma_{\mathrm{n}} \mathrm{y}\right) \cos \left(v_{\mathrm{p}} \mathrm{z}\right)}{\gamma_{\mathrm{n}}\left(\gamma_{\mathrm{n}}{ }^{2}+v_{\mathrm{p}}^{2}\right)}\left(1-\mathrm{e}^{-\alpha\left(\gamma_{\mathrm{n}}^{2}+v_{\mathrm{p}}^{2}\right) \mathrm{t}}\right)+ \\
& \left.4 \sum_{\mathrm{p}=1}^{\infty} \sum_{\mathrm{n}=1}^{\infty} \sum_{\mathrm{m}=1}^{\infty} \frac{\mathrm{J}_{\mathrm{m}} \mathrm{K}_{\mathrm{n}} \cos \left(\beta_{\mathrm{m}} \mathrm{x}\right) \cos \left(\gamma_{\mathrm{n}} \mathrm{y}\right) \cos \left(v_{\mathrm{p}} \mathrm{z}\right)}{\beta_{\mathrm{m}} \gamma_{\mathrm{n}}\left(\beta_{\mathrm{m}}{ }^{2}+\gamma_{\mathrm{n}}{ }^{2}+v_{\mathrm{p}}{ }^{2}\right)}\left(1-\mathrm{e}^{-\alpha\left(\beta_{\mathrm{m}}^{2}+\gamma_{\mathrm{n}}^{2}+v_{\mathrm{p}}^{2}\right) \mathrm{t}}\right)\right\} \\
& \text { where : } \beta_{m}=\frac{m \pi}{A}, \gamma_{n}=\frac{n \pi}{B}, v_{p}=\frac{(p-0.5) \pi}{C}, m, n, p=1,2,3, . . \\
& \mathrm{J}_{\mathrm{m}}=\sin \left(\beta_{\mathrm{m}} \mathrm{A}_{2}\right)-\sin \left(\beta_{\mathrm{m}} \mathrm{A}_{1}\right), \mathrm{K}_{\mathrm{n}}=\sin \left(\gamma_{\mathrm{n}} \mathrm{B}_{2}\right)-\sin \left(\gamma_{\mathrm{n}} \mathrm{B}_{1}\right) \\
& \mathrm{Z}_{\text {th }, \text { STGF }}(\mathrm{r}, \mathrm{t})=\frac{\alpha}{4 \mathrm{~kL}_{\mathrm{x}} \mathrm{L}_{\mathrm{x}}} \int_{0}^{\mathrm{t}}\left\{(4 \pi \alpha \mathrm{u})^{-\frac{1}{2}} \sum_{\mathrm{m}=-\infty}^{\infty} \sum_{\mathrm{n}=-\infty}^{\infty} \sum_{\mathrm{p}=-\infty}^{\infty}(-1)^{\mathrm{p}} \times 2 \mathrm{e}^{-\frac{(2 \mathrm{pC}+\mathrm{z})^{2}}{4 \alpha \mathrm{u}}}\right. \\
& \left.\left[t_{1}\left(-A_{1}\right)-t_{1}\left(-A_{2}\right)+t_{1}\left(A_{2}\right)-t_{1}\left(A_{1}\right)\right]\left[t_{2}\left(-B_{1}\right)-t_{2}\left(-B_{2}\right)+t_{2}\left(B_{2}\right)-t_{2}\left(B_{1}\right)\right]\right\} d u \\
& \text { where } \mathrm{t}_{1}(\mathrm{a})=\operatorname{erf}\left(\frac{2 \mathrm{~mA}+\mathrm{x}+\mathrm{a}}{\sqrt{4 \alpha u}}\right), \mathrm{t}_{2}(\mathrm{~b})=\operatorname{erf}\left(\frac{2 \mathrm{nB}+\mathrm{y}+\mathrm{b}}{\sqrt{4 \alpha u}}\right)
\end{aligned}
$$

\subsection{Efficiency of the LTGF Solution}

To check the reliability of the solution, the thermal impedance $\mathrm{Z}_{\mathrm{th}}(\mathrm{t})$ has been calculated for the center of centered SHS. The domain is described by $A=B=175 \mu \mathrm{m}$, $\mathrm{C}=100 \mu \mathrm{m}$ and $\mathrm{L}_{\mathrm{x}}=\mathrm{L}_{\mathrm{y}}=5 \mu \mathrm{m}$ but due to the symmetry of the problem, only its quarter is considered to improve the efficiency of the calculation procedure. The different number of terms has been used and four sets of sum limits listed in Table 1 were considered. The number of terms for the single sum over $(p)$ index is $Q_{1}$, for the double sums over $(m, p)$ and $(n, p)$ indexes it equals $\mathrm{M}_{2} \cdot \mathrm{Q}_{2}+\mathrm{N}_{3} \cdot \mathrm{Q}_{3}$, and for the triple sum it is $\mathrm{M}_{4} \cdot \mathrm{N}_{4} \cdot \mathrm{Q}_{4}$. The total number of terms $\mathrm{N}_{\text {TOT }}$ is then a sum of these products. 
Table 1. Used upper sum limits.

\begin{tabular}{c|cccc}
\hline No. & 1 & 2 & 3 & 4 \\
\hline $\mathrm{P}_{1}$ & 225 & 708 & 2237 & 7072 \\
$\mathrm{M}_{2}, \mathrm{~N}_{3}$ & 112 & 239 & 511 & 1097 \\
$\mathrm{P}_{2}, \mathrm{P}_{3}$ & 215 & 469 & 1013 & 2158 \\
$\mathrm{M}_{4}$ & 83 & 146 & 258 & 457 \\
$\mathrm{~N}_{4}$ & 83 & 146 & 258 & 457 \\
$\mathrm{P}_{4}$ & 160 & 286 & 510 & 908 \\
\hline $\mathrm{N}_{\text {tot }}$ & $\mathbf{1 . 1}$ & $\mathbf{6 . 3}$ & $\mathbf{3 5}$ & $\mathbf{1 9 4}$ \\
{$[\mathbf{1 e}+\mathbf{6}]$} & & &
\end{tabular}

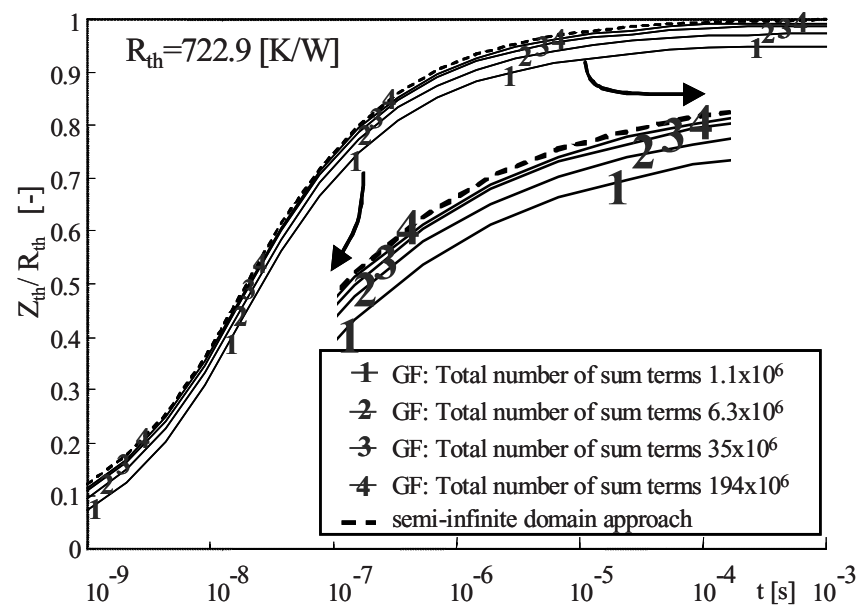

Fig. 2. Normalized thermal impedance affected by the truncation error for different number of terms (in the table) using LTGF approach. The heat source is in the center of the domain

For small times, when the heat diffusion does not reach the side walls, the impedance curves should fit the semi-infinite one but it is not observed. If more terms are included, the thermal impedance tends to fit this curve, but even a large number of terms like $2 \times 10^{8}$ is not sufficient to make the error negligible. To find the most accurate results, 6 hours were needed to compute 20 time points on a $660 \mathrm{MHz} \mathrm{PC}$ computer.

In Fig.2, the curves 1-4 are parallel and the difference between them is constant and does not depend on the time. It means that the error resulting from the series limitation is not a function of time. It is possible in such a case only when the convergence of the time dependent terms is much faster than the convergence of the time independent ones. This remark allows considering the full solution as the sum of the slow converging steady state solution $R_{t h}(r)$ and the time dependent part $\Gamma(r, t)$ that converges faster due to covering an exponential function of time [7].

$$
\mathrm{Z}_{\text {th }, \text { LTGF }}(\mathrm{r}, \mathrm{t})=\mathrm{R}_{\mathrm{th}}(\mathrm{r})+\Gamma(\mathrm{r}, \mathrm{t})
$$

\subsection{Efficiency of the STGF Solution}

The STGF solution (7) has a form of triple series covering integrals with respect to time what means that a numerical integration must be performed. The terms of the series correspond to image sources that are used in infinite domain to set proper boundary conditions. In used formalism each term correspond to 8 image sources. The solution for semi-infinite domain [6] can be also achieved from (7) by taking $(\mathrm{m}, \mathrm{n}, \mathrm{p})=(0,0,0)$ and neglecting some of the remaining elements. 


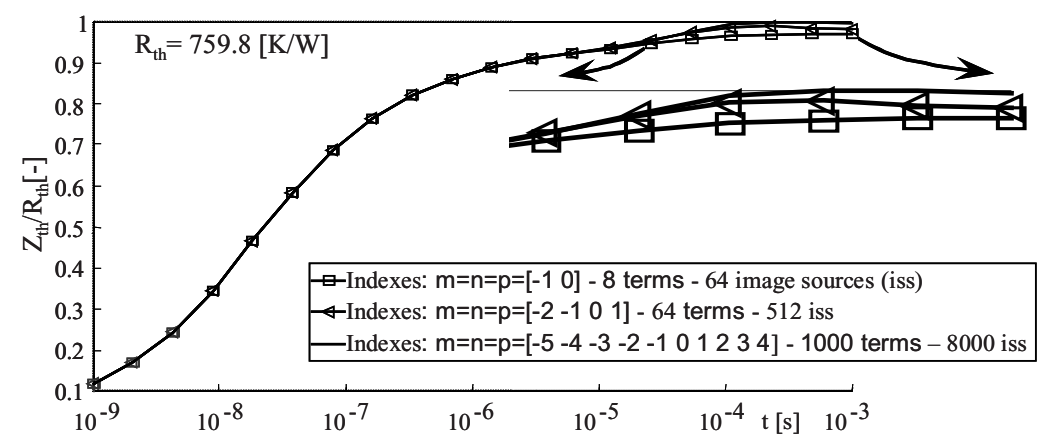

Fig. 3. Normalized thermal impedance for different number of terms. The heat source is in the center of the domain

To investigate the properties of STGF solution, as previous, the thermal impedance in the middle of the centered heat source has been calculated utilizing different number of terms and the obtained curves are depicted in Fig. 3. The dimensions of the domain were fixed as follows: $A=B=90 \mu \mathrm{m}, \mathrm{C}=100 \mu \mathrm{m}, \mathrm{L}_{\mathrm{x}}=\mathrm{L}_{\mathrm{y}}=5 \mu \mathrm{m}$.

In comparison with the LTGF approach, the truncation error of the STGF solution is not constant with time. The best agreement is found for the small times. When the time increases, the discrepancy appears. It can be explained by considering the terms as related to the image sources. For small times a few terms (i.e. images) are necessary only to set proper boundary conditions. When the time increases more terms should be utilized. If number of terms is too small, the boundary conditions are not well established and the error appears. In the example even 1000 terms (8000 image sources) were not enough to make the error unnoticed for $\mathrm{t}=10^{-3} \mathrm{~s}$.

Compared to LTGF approach, the STGF solution involves an integral over time. It has to be obtained numerically. Thus, although the number of terms is much lower than for the LTGF, the numerical integration is an additional operation that must be performed carefully (due to singularity for $\mathrm{u}=0$ ) so as no essential additional error related to integration process were introduced. The need of the numerical integration decreases the efficiency of the STGF approach that has better numerical performance for small times.

\section{The Time Partitioning Approach}

For small times very few terms of the STGF solution can be enough to get the satisfactory accuracy. In some cases even a part of the final expression i.e. the part that describes the semi-finite domain solution can be sufficient. On the other hand, considering the LTGF solution, the larger time is the lower number of terms needs to be included to obtain exact value of the transient part $\Gamma(\mathrm{r}, \mathrm{t})$ of the full solution. The time partitioning approach [4] tries to combine these features: for times lower than some value $t_{p}\left(t<t_{p}\right)$ the STGF solution is used and if the solution for $t>t_{p}$ is needed, the impedance is calculated as: 


$$
\mathrm{Z}_{\text {th }}\left(\mathrm{r}, \mathrm{t}>\mathrm{t}_{\mathrm{p}}\right)=\mathrm{Z}_{\mathrm{th}, \mathrm{STGF}}\left(\mathrm{r}, \mathrm{t}_{\mathrm{p}}\right)+\Gamma(\mathrm{r}, \mathrm{t})-\Gamma\left(\mathrm{r}, \mathrm{t}_{\mathrm{p}}\right)
$$

It means that $\mathrm{Z}_{\mathrm{th}, \mathrm{STGF}}\left(\mathrm{r}, \mathrm{t}_{\mathrm{p}}\right)$ is calculated using the STGF approach for the $\mathrm{t}_{\mathrm{p}}$ point and the numerical integration limited to the range from 0 to $t_{p}$. To complete the thermal impedance to its value at point $t$, the formula for LTGF approach is used. In this case, however, its transient parts calculated for the while $t_{p}$ and $t$ are necessary only since the difference $\Gamma(\mathrm{r}, \mathrm{t})-\Gamma\left(\mathrm{r}, \mathrm{t}_{\mathrm{p}}\right)$, corresponds to the thermal impedance increase from $\mathrm{t}_{\mathrm{p}}$ to t. In the time partitioning solution (9), there is no slow convergent steady state part of the LTGF solution and the STGF method is used only up to $t_{p}$ time point. The $t_{p}$ time can be chosen arbitrarily to optimize the speed of the evaluation process. The $t_{p}$ must be low enough to use as few as possible image sources since numerical integration is necessary. On the other hand the larger $t_{p}$ is the less terms are used by $\Gamma(r, t)$.
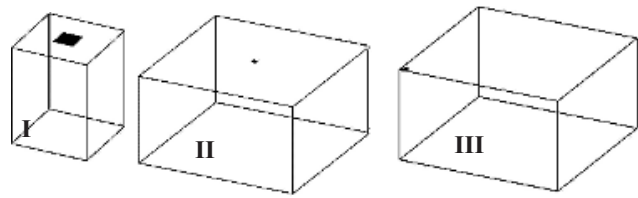

Fig. 4. Domains used to compare the efficiency. $C=100 \mu \mathrm{m}$ I: $L_{x}=L_{y}=23 \mu \mathrm{m}, A=B=90 \mu \mathrm{m}$, II: $\mathrm{L}_{\mathrm{x}}=\mathrm{L}_{\mathrm{y}}=5 \mu \mathrm{m}, \mathrm{A}=\mathrm{B}=175 \mu \mathrm{m}, \mathrm{III}: \mathrm{L}_{\mathrm{x}}=\mathrm{L}_{\mathrm{y}}=5 \mu \mathrm{m}, \mathrm{A}=\mathrm{B}=175 \mu \mathrm{m}$, the center of the heat source (leftdown corner of the domain) is at $(5,5) \mu \mathrm{m}$ point. The domains are in scale. The heat sources can be seen as small black rectangles on the top surface

To check the efficiency of the time partitioning approach the thermal impedance has been evaluated at the source centers for 3 test domains (Fig. 4) at 20 time points from $1 \mathrm{~ns}$ to $1 \mathrm{~ms}$ in logarithmic scale. Two solutions are considered:

- $\quad$ the standard LTGF solution (Eq. (6))

- $\quad$ the time partitioning method (Eq. (9)).

To make the results comparable, the maximal relative error was made for both methods equal to $6 \%$.

All simulations were done in Matlab6.5 environment using the quadl.m procedure for numerical integration, which employs the Gauss-Lobatto quadrature algorithm. The results of calculation are summarized in Table 2 that covers the total time of calculations (ToC), the total number of terms for the LTGF solution (NT) and the time partitioning point $t_{p}$. For structures I and II a quarter of the domain for the LTGF solution is used. As the STGF solution the semi-infinite domain solution is used only. The $t_{p}$ is chosen to be latest available time point where the semi-infinite domain solution is valid for the finite domain.

The results presented in the table show that proper choice of the solution formulae can speed up calculations from $6 \mathrm{~h}$ to $0.1 \mathrm{~s}$ that is more than $1 \cdot 10^{5}$ times. The ToC for the time partitioning method can be optimised by the proper choice of the $t_{p}$ point. 
Table 2. Comparison of efficiency of analyzed methods

\begin{tabular}{|c|c|c|c|}
\hline $\begin{array}{l}\text { Doma } \\
\text { Method }\end{array}$ & ain & II & III \\
\hline $\mathrm{i}$ & $\Gamma \mathrm{To}=25 \mathrm{~min}, \mathrm{NT}=107 \times 106$ & $\underline{\mathrm{ToC}}=6 \mathrm{~h}(660 \mathrm{MHz} P C), \mathrm{NT}=3880 \times 106$ & - \\
\hline$\frac{\mathrm{Tc}}{\mathrm{tp}_{\mathrm{p}}}$ & 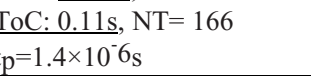 & $\frac{\text { ToC: } 0.15 \mathrm{~s}, \mathrm{NT}}{\mathrm{tp}_{\mathrm{p}}=4.8 \times 10^{-4} \mathrm{~s}_{\mathrm{s}}}=8$ & $\frac{\text { ToC: } 0.7 \mathrm{~s}, \mathrm{NT}}{\mathrm{t}_{\mathrm{p}}=1.8 \times 10^{-} 8 \mathrm{~s}}=105$ \\
\hline
\end{tabular}

\section{Conclusions}

The electro-thermal analysis of any device, especially ICs, becomes indispensable and the ICs should be considered in their thermal domain as 3D systems. Describing the electrical behavior of the cell or cluster by simple model as it has been presented, the analytical solutions of the 3D heat transfer phenomenon seems to be well suited in this case. Although different analytical formulas are available, evaluation process of the expressions may involve a lot of computational effort and its efficiency is changes depending on the chosen analytical solution and particular conditions. The LTGF and the STGF approaches have serious drawbacks when considered separately. But both solutions can be combined in time partitioning method. Although this idea has been indicated by Beck et. al. in [4], on the best knowledge of authors, it has not been used in any application. Presented analysis shows that the time partitioning approach, as a proper combination of methods, may improve the efficiency by few orders of magnitude.

\section{References}

1. d'Alessandro, V., Rinaldi, N.: A critical review of thermal models for electrothermal simulations. Solid-State electronics, Vol. 46, (2002) 487-496

2. d'Alessandro, V., Rinaldi, N., Spirito, P.: Thermal modeling and simulation of multicellular power devices. Proc. MicroTherm 2003, (Łód 2003, Poland).

3. Batty, W., Christoffersen, C. E., Panks, A. J., David, S., Snowden, C. M., Steer, M. B.: Electrothermal CAD of power devices and circuits with fully physical time-dependent compact thermal modeling of complex nonlinear 3-D systems. IEEE Trans. on Components and Packaging Technologies, Vol. 24, (2001) 566-590

4. Beck, J.V., Cole K., Hadji-Sheikh A., Litkouhi B.: Heat conduction using Green's functions. Hemispherev Publishing Corp., Washington DC (1992)

5. Macchiaroli, M., Rinaldi, N., d'Alessandro, V., Breglio, G., Spirito, P.: A new Electrothermal Simulation Tool for the Analysis of Bipolar Devices and Circuits. Proc. $7^{\text {th }}$ Thermal Investigations of ICs and systems (THERMINIC) Workshop, (Paris 2001) 281-286.

6. Rinaldi, N., On the modeling of the transient thermal behavior of semiconductor devices IEEE Trans. on Electron devices, Vol. 48, 2796-2802 (2001)

7. Wo ny, J., Rinaldi, N., Owczarek, M., Nowak, J.: Optimizing The Numerical Procedure of Temperature Evaluating from The Heat Diffusion Equation. Accepted for TCSET conference, Lviv (2004). 